INVESTIGACIONES

\title{
Sexo y edad del alumnado sobre las orientaciones de meta en la Educación Física
}

\author{
Sex and age of the students on goal orientations and motivation in Physical Education \\ Sexo e idade dos alunos sobre as orientações de meta em Educação Física
}

\author{
Baena-Extremera, Antonio ${ }^{a}$; Granero-Gallegos, Antonio ${ }^{b}$

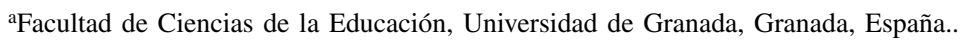 \\ Teléfono: 958242829. Correo electrónico: abaenaextrem@ugr.es \\ bFacultad de Ciencias de la Educación, Universidad de Almería, Almería, España. \\ Teléfono: 00950015073. Correo electrónico: agranero@ual.es
}

\begin{abstract}
RESUMEN
El objetivo de este trabajo fue conocer los efectos de interacción de sexo y edad sobre las orientaciones de meta, motivación autodeterminada y clima motivacional en Educación Física (EF). Participaron 1.298 estudiantes de secundaria de las provincias de Almería, Granada y Málaga, con edades entre 12 y 19 años. Como instrumentos se utilizaron las versiones españolas y adaptadas a la EF de: Cuestionario de Percepción de Éxito, Cuestionario de Orientación al Aprendizaje y al Rendimiento en las Clases de Educación Física y Escala de Motivación en el Deporte. Se realizaron análisis descriptivos y multivariantes (MANOVA 2x3). Los resultados muestran diferencias significativas por sexo y edad. Se concluye que el profesor debe diseñar sus clases con un clima motivacional orientado a la tarea, favoreciendo un entorno ideal para la mejora del aprendizaje y el incremento del compromiso del alumnado en las clases EF.
\end{abstract}

Palabras clave: motivación, tarea, ego, educación física, profesor, adolescencia.

\begin{abstract}
The aim of this study was to determine the effects of interaction between sex and age in relation to goal orientation, motivational environment, and self-determined motivation in physical education (PE) of 1298 high school students from the provinces of Almería, Granada and Málaga, aged between 12 and 19 years. As instruments we used the Spanish version adapted to the PE: Perception of Success Questionnaire, Learning and Performance Orientations in Physical Education Classes Questionnaire and Sport Motivation Scale; as well as, the descriptive and multivariate analyzes (MANOVA 2x3). Results show significant differences based on gender and age. We conclude that the teacher should design his/her classes with a task-oriented motivational environment, granting an ideal environment for improved learning and an increased engagement of students in PE classes.
\end{abstract}

Key words: motivation, task, ego, physical education, teacher, adolescence.

\section{RESUMO}

Objetivou conhecer os efeitos de interação de sexo e idade sobre as orientações de meta, motivação autodeterminada e clima motivacional em aulas de Educação Física (EF). Participaram um total de 1.298 estudantes da Educação Secundária Obrigatória das províncias de Almería, Granada e Málaga, com idades entre 12 e 19 anos. Como instrumentos, utilizaram-se as versões em espanhol, adaptadas à EF do Questionário de Percepção de Exxito, Questionário de Orientação para a Aprendizagem e Desempenho em Educação Física e Escala de Motivação no esporte. Realiaram-se análises descritivas e multivariantes (MANOVA 2x3). Resultados mostram diferenças significativas por sexo e idade. Conclui-se que o professor deve planejar suas aulas com um clima motivacional voltado para a tarefa, favorecendo um lugar ideal para a melhora da aprendizagem e o aumento do compromisso dos estudantes nas aulas de EF.

Palavras chave: motivação, tarefa, ego, educação física, professor, adolescência. 


\section{INTRODUCCIÓN}

En la legislación educativa española (Real Decreto 1631/2006 de enseñanzas mínimas para la Educación Secundaria Obligatoria) la materia de educación física (EF) en la educación secundaria obligatoria (ESO) debe contribuir a desarrollar las capacidades instrumentales y a generar hábitos de práctica continuada de actividad física. Según este Real Decreto (2006: 710):

(...) a través del ejercicio físico se contribuye a la conservación y mejora de la salud y el estado físico, a la prevención de determinadas enfermedades y disfunciones y al equilibrio psíquico, en la medida en que las personas, a través del mismo liberan tensiones, realizan actividades de ocio, y disfrutan de su propio movimiento y de su eficacia corporal. Todo ello resulta incluso más necesario dado su papel para compensar las restricciones del medio y el sedentarismo habitual de la sociedad actual. La materia de Educación física actúa en este sentido como factor de prevención de primer orden.

Para conseguir este reto es necesario que el alumnado reciba clases que propicien esta situación, que se les motive y despierte el interés por adquirir estas capacidades instrumentales y mejorar sus hábitos de práctica físico-deportiva. Para ello, y como demuestran algunos trabajos (Moreno, Trigueros y Rivera, 2013; Moreno, Zomeño, Marín, Ruiz y Cervelló, 2013; Jiménez, Cervelló, García, Santos e Iglesias, 2007; Trudeau, Laurencelle, Tremblay, Rajic \& Shephard, 1999), la figura del docente es clave en la creación de un ambiente de clase y de un clima que motive a la participación en actividades físicas y deportivas dentro y fuera de la escuela (Moreno, Vera y Cervelló, 2006d).

Para estudiar la motivación y los factores que dependen de ella en el alumnado adolescente se han seguido dos de las teorías motivacionales más utilizadas en la actualidad: Metas de Logro (Nicholls, 1989) y Autodeterminación (Deci \& Ryan, 2000, 1985; Ryan \& Deci, 2000).

La primera de ellas tiene como objetivo el análisis de los diferentes factores disposicionales y ambientales que influyen en la motivación de logro del deportista. Esta teoría sostiene que las personas se implican en los contextos de logro con la finalidad de demostrar competencia o habilidad (Cervelló y Santos-Rosa, 2000). Para el alumno, las clases de EF son un contexto de exigencia de logro (Moreno et al., 2006d), ya que suelen buscar en ella demostrar su habilidad. A pesar de ello, este nivel de competencia o habilidad dependerá de la percepción subjetiva de éxito o fracaso del alumno (Nicholls, 1989). La adopción de estos criterios depende tanto de las características personales (orientación disposicional) como de aspectos sociales y situacionales (clima motivacional).

Según este constructo teórico existen dos tipos de orientaciones de meta disposicionales, por un lado la orientación a la tarea que se manifiesta cuando la meta se dirige al aprendizaje y los practicantes juzgan su nivel de capacidad mediante un proceso de comparación con ellos mismos y, por otro lado, la orientación al ego, donde la meta se caracteriza por la competitividad y los practicantes juzgan su nivel de competencia en referencia a los demás. Como se puede apreciar, sería muy interesante conocer por parte del profesor las orientaciones de meta de nuestros alumnos, de cara a posibles actuaciones docentes. Pero para diseñar actuaciones docentes previamente habría que preguntarse ¿son iguales las orientaciones de meta en los chicos que en las chicas? En los estudios realizados por Castillo, Balaguer y Duda (2000) y Duda \& Whitehead (1998) se muestra que 
los varones reflejan mayor preocupación por la victoria y por demostrar sus capacidades en ambientes de logro manifestando, por tanto, una mayor orientación al ego que ellas.

Por otro lado se encuentra el clima motivacional, el que se crea a partir de las estructuras creadas por el entorno que rodea al alumno (familia, profesores e iguales) y que va a ser percibido por ellos (Llanos, Cervelló, y Tabernero, 2008). Este clima motivacional se diferencia en función del criterio de éxito establecido, según esté orientado a la tarea o al ego (Ames, 1992a). Aunque los compañeros de clase influyen en el clima motivacional que percibe el alumno, es el profesor el que tiene un rol fundamental en la creación del clima motivacional de clase. Un clima orientado a la tarea es aquel donde el profesor fomenta la autonomía, la autodirección, la participación, el dominio de la tarea a nivel individual, la resolución de problemas y las mismas oportunidades de recibir recompensas. En cambio, en un clima orientado al ego el docente controla mucho más la dinámica de la clase, fomenta competiciones interpersonales, evaluaciones públicas y suele recompensar a los estudiantes mejor dotados (Roberts, 2001; Cervello y Santos-Rosa, 2000; Nicholls, 1989). De ahí que aquellos alumnos que perciben un clima motivacional orientado a la tarea consideren la EF como un fin en sí mismo y se diviertan mucho más que aquellos con un clima orientado al ego, estos consideran las clases como un medio para conseguir otras cosas (Cervelló, Escartí y Balagué, 1999).

Utilizando ambos constructos, resaltar que existe una relación positiva y significativa entre el clima motivacional y la orientación de metas. El clima motivacional orientado a la tarea derivará en una orientación de metas orientada a la tarea, mientras que un clima orientado al ego derivará en una orientación al ego (Llanos et al., 2008; Jiménez et al., 2007; Sproule, Wang, Morgan, McNeill \& McMorris, 2007; Cervello y Santos-Rosa, 2000; Ames, 1992a, 1992b; Duda \& Nicholls, 1992).

Son muchos los estudios que han demostrado que el sexo es una variable moduladora del clima motivacional percibido por el alumno (Jairo y Márquez, 2008). Los varones muestran normalmente puntuaciones más altas que las mujeres, en el clima motivacional orientado al ego (Jairo y Marquez, 2008; Carr y Weigand, 2001; Cervello y Santos-Rosa, 2000), mientras que las mujeres están más implicadas en un clima motivacional orientado a la tarea (Ntoumanis \& Biddle, 1999; Walling, Duda \& Chi, 1993).

La segunda de las teorías motivacionales que se utilizó es la teoría de la Autodeterminación (SDT) (Deci \& Ryan, 1985), la que establece diferentes niveles en los deportistas. De este modo, ordenados de mayor a menor grado de autodeterminación, la conducta del deportista puede estar intrínsecamente motivada, extrínsecamente motivada o amotivada hacia la práctica deportiva.

Aplicando esta teoría a la EF, el alumnado que participa en clase porque disfruta aprendiendo y vivenciando las diferentes prácticas, probablemente reflejaría una motivación intrínseca, que es la forma de motivación más autodeterminada (González-Cutre, Sicilia y Moreno, 2011). El deportista se involucra en las actividades que le interesan y se compromete en ellas libremente, gracias al placer y al disfrute que obtiene practicando (Deci \& Ryan, 2000, 1985). Según esta teoría, existen tres manifestaciones diferentes de motivación intrínseca: motivación por el conocimiento, por el logro y por el estímulo que da la experiencia o hacia la estimulación (Vallerand, Blais, Brière et Pelletier, 1989). En la motivación por el conocimiento, el estudiante se compromete en las clases de EF por placer y satisfacción mientras intenta aprender. La motivación hacia el logro muestra al alumno que se compromete en las clases por placer mientras intenta mejorar o superarse a sí mismo. 
Y la motivación intrínseca hacia la estimulación refleja al alumno que se compromete en las clases con la finalidad de experimentar sensaciones asociadas a sus propios sentidos.

En el lado opuesto se encuentra la motivación extrínseca, que proviene de fuentes externas. Según Deci \& Ryan (2000, 1985) se identifican cuatro tipos diferentes de motivación extrínseca. Ordenados de mayor a menor grado de autodeterminación encontramos la regulación integrada, la regulación identificada, la introyectada y la regulación externa. En cuanto a la regulación integrada, el alumno pone en congruencia valores y formas de concebir el mundo. Es la forma más autodeterminada de regulación interiorizada y casi no puede distinguirse de la motivación intrínseca. Sigue siendo una forma de motivación extrínseca, ya que el discente no actúa por el placer inherente de la actividad (Ryan \& Deci, 2000). La regulación identificada refleja que el alumno conoce la importancia de la EF y los beneficios que aporta y por eso realiza la práctica. La regulación introyectada muestra al estudiante que practica en la clase por culpabilidad. Y en la regulación externa, el alumno participa en las clases porque tiene que hacerlo, debido a las normas educativas, evitando así un posible castigo (González-Cutre et al., 2011).

Por último se encuentra la amotivación, en la que el deportista no se encuentra motivado ni intrínseca ni extrínsecamente (Pelletier, Vallerand, Green-Demers, Brière \& Blais, 1995b); es decir, se caracteriza porque el deportista no tiene la intención de realizar algo (Ryan \& Deci, 2000). Se trataría de aquel alumno que no entiende por qué hay que tener clases de EF, ni conoce para qué sirve, por lo que ve esta asignatura como una pérdida de tiempo (González-Cutre et al., 2011).

Resaltar que son muchos los estudios que han comprobado las relaciones existentes entre la teoría de las metas de logro y la teoría de la Autodeterminación. Incluso existen estudios que han demostrado que existe una relación significativa entre las orientaciones de meta adoptadas por el alumnado, el clima motivacional percibido, la motivación y la intención de ser activo en el futuro (Sproule et al., 2007; Ntoumanis, 2005; Hein, Müür \& Koka, 2004).

Como se puede apreciar, el interés que despierta para el profesor de EF conocer las orientaciones, el clima y la motivación de sus alumnos es muy notable, pues a partir de este conocimiento, se podrían establecer propuestas didácticas que ayuden a adquirir no solo las capacidades instrumentales, sino también los hábitos de práctica física. Por todo ello, el objetivo de este trabajo ha sido conocer los efectos de interacción del sexo y la edad del alumnado sobre las orientaciones de meta, motivación autodeterminada y clima motivacional en EF.

\section{MÉTODO}

\subsection{PARTICIPANTES}

La selección de la muestra fue de tipo no probabilístico y por conveniencia, según los sujetos a los que se pudo acceder. Participaron 1.298 estudiantes (626 varones $=48,2 \%$; 672 mujeres $=51,8 \%$ ) de ocho centros de secundaria de las provincias de Almería, Granada y Málaga. El rango de edad estuvo comprendido entre 12 y 19 años $(M=15,13 ; D T=$ $1,43)$, siendo la edad media de los chicos $15,26(D T=1,42)$, y la de las chicas 15,01 $(D T=1,43)$. El $28,1 \%$ del alumnado fue del $1^{\text {er }}$ ciclo de ESO, el $41,87 \%$ de $2^{\circ}$ ciclo de ESO y $29,4 \%$ de bachillerato (enseñanza secundaria postobligatoria). 


\subsection{INSTRUMENTOS}

Cuestionario de Percepción de Éxito (POSQ). Se utilizó la versión en español (Cervelló et al., 1999) adaptada a la EF (Granero-Gallegos, Baena-Extremera, Gómez-López y Abraldes, 2014) del original Perception of Success Questionnaire (Roberts, Treasure \& Balague, 1998; Roberts \& Balague, 1991). Consta de 12 ítems para medir las orientaciones de meta disposicionales del alumnado en las clases de EF, mediante dos dimensiones que miden la orientación hacia la tarea (6 ítem) y la orientación hacia el ego (6 ítems). Las respuestas se recogieron en una escala de ítems politómicos de cinco puntos que oscila desde mиy en desacuerdo (1) a muy de acuerdo (5). En el presente estudio la consistencia interna de la subescala orientación hacia la tarea fue de alfa de Cronbach $(\alpha)=0,87$ y la de orientación hacia el ego, $\alpha=0,92$.

Cuestionario de Orientación al Aprendizaje y al Rendimiento en las Clases de Educación Física (LAPOPECQ). Se utilizó la versión española (Cervelló et al., 2002) del original Learning and Performance Orientations in Physical Education Classes Questionnaire (Papaioannou, 1994). Esta escala mide la percepción de los estudiantes del clima motivacional en las clases de EF. Se compone por 27 ítems y posee dos dimensiones: Percepción del clima motivacional que implica al aprendizaje (clima al aprendizaje; 13 ítems) y Percepción del clima motivacional que implica al rendimiento (clima al rendimiento; 14 ítems). Las respuestas se recogieron en una escala de ítems politómicos con rango de puntuación entre 0 (totalmente en desacuerdo) y 10 (totalmente de acuerdo). En el presente estudio la consistencia interna de la subescala clima implicante al aprendizaje fue $\alpha=0,91$ y la de clima implicante al rendimiento, $\alpha=0,88$.

Escala de Motivación en el Deporte (SMS). Se empleó la versión validada al castellano por Núñez et al. (2006) y adaptada a la EF (Granero-Gallegos y Baena-Extremera, 2013). La escala original se denominó Échelle de Motivation dans les Sports (EMS) (Brière, Vallerand, Blais et Pelletier, 1995) y fue traducida al inglés por Pelletier et al. (1995a) pasando a denominarse Sport Motivation Scale (SMS). Consta de 28 ítems que medían los diferentes tipos de motivación establecidos por la teoría de la autodeterminación (Deci \& Ryan, 1985) que sugiere la explicación multidimensional de la motivación: desmotivación (4 ítems), motivación extrínseca (ME) (12 ítems; regulación externa, introyectada e identificada), y motivación intrínseca (MI) (12 ítems; al conocimiento, al logro y estimulación); en total corresponden cuatro ítems a cada factor. Las respuestas fueron recogidas en una escala de ítems politómicos, con un rango de puntuación que oscilaba entre 1 (totalmente en desacuerdo) a 7 (totalmente de acuerdo). La consistencia interna hallada en el presente estudio fue: MI al conocimiento, $\alpha=0,84 ;$ MI hacia el logro, $\alpha=0,82 ;$ MI hacia la estimulación, $\alpha=0,82 ;$ ME identificada, $\alpha=0,80 ; M E$ introyectada, $\alpha=0,69 ; M E$ de regulación externa, $\alpha=0,77$; y amotivación, $\alpha=0,72$. Aunque algunos factores obtuvieron un valor de consistencia interna inferior a 0,70 (pero entre 0,60 y 0,70), se puede considerar marginalmente aceptable (Taylor, Ntoumanis \& Standage, 2008) debido al pequeño número de ítems de la subescala.

\subsection{PROCEDIMIENTO}

Se obtuvo permiso para realizar la investigación por parte de los órganos competentes, tanto de los centros educativos de secundaria como universitarios. Se informó, en 
detalle, a padres/tutores y adolescentes acerca del protocolo y objeto del estudio. La firma del consentimiento informado por parte de ambos fue requisito indispensable para poder participar. Los instrumentos para medir las diferentes variables se administraron en el aula por los propios investigadores y sin la presencia del docente. Todos los participantes fueron informados del objetivo de estudio, voluntariedad y confidencialidad de las respuestas y manejo de datos, que no había respuestas correctas o incorrectas, pidiéndoles al comienzo de este que contestaran con la máxima sinceridad y honestidad.

\subsection{ANÁLISIS ESTADÍSTICO}

Los análisis de ítems, homogeneidad, consistencia interna de cada subescala (alfa de Cronbach), análisis multivariante de la varianza 2 (sexo) x 3 (edad) se realizaron con el SPSS v.17. Se calcularon los estadísticos descriptivos y los índices de asimetría y curtosis, siendo, en general, próximos a cero y <2,0, como recomiendan Bollen \& Long (1994). Se evaluó la estructura factorial de cada instrumento con análisis factorial confirmatorio (AFC) realizado con LISREL 8.80.

\subsection{PROPIEDADES PSICOMÉTRICAS DE LOS INSTRUMENTOS}

Debido a que las estructuras que subyacen en cada instrumento analizado han sido consistentemente determinadas en la literatura, para evaluar la estructura factorial de cada escala se realizó un AFC. Se utilizó el método de estimación weighted least squares (WLS) para variables ordinales del programa LISREL 8.80 (Jöreskog \& Sörbom, 1993), como input para el análisis de datos se utilizó la matriz de correlaciones policóricas y la matriz de covarianzas asintóticas. Se asumió la existencia de las variables latentes según los instrumentos originales descritos.

Teniendo en cuenta las recomendaciones que desaconsejan utilizar una única medida de ajuste global del modelo (Bentler, 2007), cada modelo fue evaluado con una combinación de índices de ajuste absolutos y relativos. Entre los absolutos, se utilizó el valor $p$ asociado con el estadístico chi cuadrado $\left(\chi^{2}\right)$. La ratio entre $\chi^{2}$ y grados de libertad $(g l)$ $\left(\chi^{2} / g l\right)$ es un heurístico que se utiliza para reducir la sensibilidad del $\chi^{2}$ al tamaño de la muestra. En un modelo perfecto su valor sería 1.0; las ratios $<2,0$ se considerarán como indicadores de muy buen ajuste del modelo (Tabachnik \& Fidell, 2006), mientras que valores $<5,0$ son considerados aceptables (Hu \& Bentler, 1999). Además, se ha calculado el GFI (índice de bondad de ajuste) y autores como Hooper, Coughlan, y Mullen (2008) consideran valores $\geq 0,95$ para un mejor ajuste del modelo. Entre los índices relativos: el NFI (índice de ajuste normalizado), NNFI (índice de ajuste no normativo) y CFI (índice de ajuste comparativo) y se considera que valores $\geq 0,95$ indican un buen ajuste (Hu y Bentler, 1999). Autores como Kline (2005) recomiendan la utilización de RMSEA (error de aproximación cuadrático medio) y, según Hu y Bentler (1999) un valor $\leq 0,06$ indicaría un buen ajuste. Los parámetros estimados se consideran significativos cuando el valor asociado al valor $t>1,96(p<0,05)$. Los modelos de las tres escalas presentan valores correctos que permiten determinar una aceptable bondad de ajuste del modelo original (Tabla 1). 
Tabla 1. Índices de ajuste de los modelos

\begin{tabular}{|l|c|c|c|c|c|c|c|c|c|}
\cline { 2 - 10 } \multicolumn{1}{c|}{} & \multicolumn{1}{c|}{$\boldsymbol{\chi}^{\mathbf{2}}$} & $\mathbf{g l}$ & $\mathbf{p}$ & $\chi^{\mathbf{2} / \boldsymbol{g l}}$ & $\mathbf{G F I}$ & NFI & NNFI & CFI & RMSEA \\
\hline POSQ & 95,55 & 53 & 0,001 & 1,80 & 0,99 & 0,97 & 0,98 & 0,99 & 0,04 \\
\hline LAPOPECQ & 846,61 & 323 & 0,000 & 2,62 & 0,96 & 0,97 & 0,97 & 0,95 & 0,06 \\
\hline SMS & 522,62 & 329 & 0,000 & 1,59 & 0,98 & 0,95 & 0,97 & 0,97 & 0,03 \\
\hline
\end{tabular}

Estos datos se ajustan a los parámetros establecidos, por lo que se pueden aceptar como buenos los modelos propuestos (Hu \& Bentler, 1999). Cada modelo presenta los requisitos mínimos para poder garantizar la validez convergente del mismo (Hair, Black, Babin \& Anderson, 2009): cargas factoriales estandarizadas elevadas $(>0,60)$ y estadísticamente significativas $(t$-value $>1,96)$.

\section{RESULTADOS}

\subsection{ANÁLISIS DESCRIPTIVO Y DE CORRELACIÓN}

En la Tabla 2 se exponen los valores descriptivos de cada variable. Respecto de las puntuaciones medias, en las subescalas de percepción de éxito, la orientación a la tarea obtuvo una puntuación más alta que la orientación al ego. En el clima motivacional, el clima aprendizaje obtuvo una puntuación mucho más alta que el clima rendimiento. Finalmente, en las dimensiones del SMS, los factores relacionados con la motivación intrínseca presentan valores elevados, sobre todo la MI hacia el logro; entre los valores de la motivación extrínseca destaca la puntación de la $M E$ introyectada; la menor puntuación corresponde a la amotivación.

Tabla 2. Estadísticos descriptivos de las diferentes subescalas

\begin{tabular}{|l|r|r|c|c|}
\hline \multicolumn{1}{|c|}{ Subescalas } & \multicolumn{1}{c|}{$\boldsymbol{M}$} & \multicolumn{1}{c|}{$\boldsymbol{D T}$} & Asimetría & Curtosis \\
\hline Orientación ego & 3,28 & 1,03 & $-0,25$ & $-0,53$ \\
\hline Orientación tarea & 4,20 & 1,17 & $-1,15$ & $-1,34$ \\
\hline Clima aprendizaje & 68,17 & 17,98 & $-0,21$ & $-0,54$ \\
\hline Clima rendimiento & 54,37 & 16,94 & $-0,01$ & $-0,05$ \\
\hline MI conocimiento & 4,77 & 1,36 & $-0,39$ & $-0,23$ \\
\hline MI logro & 5,04 & 1,30 & $-0,46$ & $-0,13$ \\
\hline MI estimulación & 4,83 & 1,33 & $-0,32$ & $-0,34$ \\
\hline ME identificada & 4,63 & 1,37 & $-0,26$ & $-0,45$ \\
\hline ME introyectada & 4,90 & 1,24 & $-0,41$ & $-0,08$ \\
\hline ME regulación externa & 4,15 & 1,39 & $-0,09$ & $-0,47$ \\
\hline Amotivación & 3,11 & 1,42 & 0,38 & $-0,65$ \\
\hline
\end{tabular}

${ }^{*} p<0,05 ; * * p<0,01$. 


\subsection{ANÁLISIS MULTIVARIANTE DE LA VARIANZA}

Para analizar los efectos de interacción del sexo y la edad del alumnado sobre los constructos analizados, se realizó un análisis multivariante [MANOVA 2 x 3 (sexo x edad)]; las variables independientes fueron sexo y edad, y las dependientes las subescalas del POSQ, LAPOPECQ y SMS. La homogeneidad de la covarianza se examinó mediante el test $\mathrm{M}$ de Box; se rechazó la hipótesis nula de ajuste de los datos ( $\mathrm{M}$ de Box $=484,75, F$ $=1,43, p<0,000)$. Se siguieron las sugerencias de Tabachnick \& Fidell (2006) de usar la Pillai's Trace en lugar de la Lamba de Wilks para evaluar la significación multivariada de efectos principales y de las interacciones. El contraste multivariado no demostró diferencias significativas y efectos de interacción entre las dos variables independientes (sexo x edad) (Pillai's Trace $\left.=0,02, F_{(22,2542)}=1,17, p=0,26\right)$. No obstante, se encontraron diferencias significativas en relación con el género (Pillai's Trace $=0,12 ; F_{(11,1270)}=15,14 ; p<0,000$; $d=0,12$; potencia observada $=1,0)$ y edad alumnado (Pillai's Trace $=0,06 ; F_{(22,2542)}=$ $3,69 ; p<0,000 ; d=0,03$; potencia observada $=1,0)$. Los resultados del tamaño del efecto -effect size- $(d)$ y de la potencia observada indican que las diferencias son altas. Las pruebas intersujetos mostraron diferencias muy significativas en todas las subdimensiones analizadas, excepto en el clima implicante al aprendizaje y en la amotivación.

En relación con el sexo, las pruebas de los efectos intersujetos mostraron diferencias significativas en nueve dimensiones (Tabla 3); en todos los casos, los varones presentaron valores más altos que las chicas: orientación a la tarea, orientación al ego, clima implicante al rendimiento, MI conocimiento, MI al logro, MI hacia la estimulación, ME identificada, ME introyectada y ME de regulación externa.

Tabla 3. Análisis multivariante (efectos intersujetos según sexo) en función de las subescalas del POSQ, LAPOPEQ y SMS

\begin{tabular}{|c|c|c|c|c|c|c|c|c|}
\hline & \multicolumn{2}{|c|}{$\begin{array}{c}\text { Chicos } \\
(n=626)\end{array}$} & \multicolumn{2}{|c|}{$\begin{array}{c}\text { Chicas } \\
(n=672)\end{array}$} & \multirow[b]{2}{*}{$\boldsymbol{F}$} & \multirow[b]{2}{*}{$p$} & \multirow[b]{2}{*}{$d$} & \multirow[b]{2}{*}{ Potencia observada } \\
\hline & $M$ & $D T$ & $M$ & $D T$ & & & & \\
\hline \multicolumn{9}{|l|}{ POSQ } \\
\hline Orientación ego & 3,50 & 1,01 & 3,03 & 1,03 & 56,21 & 0,000 & 0,04 & 1,00 \\
\hline Orientación tarea & 4,27 & 1,03 & 4,13 & 1,01 & 10,46 & 0,001 & 0,01 & 0,90 \\
\hline \multicolumn{9}{|l|}{ LAPOPECQ } \\
\hline Clima aprendizaje & 68,71 & 17,28 & 67,88 & 18,61 & 0,55 & 0,457 & 0,00 & 0,12 \\
\hline Clima rendimiento & 58,17 & 16,64 & 50,33 & 16,40 & 59,40 & 0,000 & 0,04 & 1,00 \\
\hline \multicolumn{9}{|l|}{ SMS } \\
\hline MI conocimiento & 4,99 & 1,30 & 4,52 & 1,38 & 32,09 & 0,000 & 0,03 & 1,00 \\
\hline MI logro & 5,26 & 1,18 & 4,80 & 1,37 & 33,60 & 0,000 & 0,03 & 1,00 \\
\hline MI estimulación & 5,08 & 1,23 & 4,56 & 1,37 & 40,98 & 0,000 & 0,03 & 1,00 \\
\hline ME identificada & 4,88 & 1,33 & 4,35 & 1,36 & 41,66 & 0,000 & 0,03 & 1,00 \\
\hline ME introyectada & 5,03 & 1,19 & 4,78 & 1,28 & 10,72 & 0,001 & 0,01 & 0,91 \\
\hline ME regulación externa & 4,52 & 1,30 & 3,78 & 1,40 & 67,66 & 0,000 & 0,05 & 1,00 \\
\hline Amotivación & 3,14 & 1,53 & 3,06 & 1,31 & 1,01 & 0,315 & 0,00 & 0,17 \\
\hline
\end{tabular}

Nota. $p$ es significativa al nivel $<0,05$. 
Según la edad del alumnado (Tabla 4), los efectos intersujetos arrojaron diferencias significativas solo en el clima implicante al rendimiento, en la $M I$ al conocimiento, $M I$ hacia la estimulación, ME identificada y ME de regulación externa. No se hallaron diferencias en las dos dimensiones de percepción de éxito. Para comprobar las diferencias entre los diferentes grupos de edad se realizaron análisis a posteriori (prueba de Bonferroni).

Tabla 4. Análisis multivariante (efectos intersujetos según edad) en función de las subescalas del POSQ, LAPOPECQ y SMS

\begin{tabular}{|c|c|c|c|c|c|c|c|c|c|c|}
\hline & \multicolumn{2}{|c|}{$\begin{array}{c}12-14 \\
(n=443)\end{array}$} & \multicolumn{2}{|c|}{$\begin{array}{c}15-16 \\
(n=638)\end{array}$} & \multicolumn{2}{|c|}{$\begin{array}{c}17-19 \\
(n=217)\end{array}$} & \multirow[b]{2}{*}{$\boldsymbol{F}$} & \multirow[b]{2}{*}{$p$} & \multirow[b]{2}{*}{$d$} & \multirow[b]{2}{*}{$\begin{array}{c}\text { Potencia } \\
\text { observada }\end{array}$} \\
\hline & $M$ & $D T$ & $M$ & $D T$ & $M$ & $D T$ & & & & \\
\hline \multicolumn{11}{|l|}{ POSQ } \\
\hline Orientación ego & 3,25 & 1,07 & 3,35 & 1,01 & 3,19 & 1,04 & 2,55 & 0,078 & 0,00 & 0,511 \\
\hline Orientación tarea & 4,21 & 1,03 & 4,21 & 1,00 & 4,17 & 1,01 & 0,26 & 0,770 & 0,00 & 0,091 \\
\hline \multicolumn{11}{|l|}{ LAPOPECQ } \\
\hline Clima aprendizaje & 69,25 & 18,22 & 67,72 & 17,56 & 67,92 & 18,72 & 0,97 & 0,379 & 0,00 & 0,219 \\
\hline Clima rendimiento & 57,03 & 17,29 & 53,66 & 16,37 & 52,01 & 17,58 & 8,21 & 0,000 & 0,02 & 0,961 \\
\hline \multicolumn{11}{|l|}{ SMS } \\
\hline MI conocimiento & 5,02 & 1,36 & 4,69 & 1,35 & 4,55 & 1,34 & 11,79 & 0,000 & 0,02 & 0,995 \\
\hline MI logro & 5,12 & 1,38 & 5,01 & 1,24 & 4,91 & 1,28 & 4,00 & 0,059 & 0,01 & 0,717 \\
\hline MI estimulación & 5,08 & 1,35 & 4,74 & 1,31 & 4,65 & 1,29 & 11,55 & 0,000 & 0,02 & 0,994 \\
\hline ME identificada & 4,90 & 1,40 & 4,54 & 1,33 & 4,40 & 1,34 & 13,23 & 0,000 & 0,02 & 0,998 \\
\hline ME introyectada & 5,02 & 1,30 & 4,86 & 1,22 & 4,83 & 1,20 & 2,60 & 0,075 & 0,00 & 0,519 \\
\hline ME regulación externa & 4,47 & 1,33 & 4,05 & 1,39 & 3,82 & 1,40 & 21,03 & 0,000 & 0,03 & 1,000 \\
\hline Amotivación & 3,16 & 1,43 & 3,11 & 1,42 & 3,02 & 1,38 & 0,73 & 0,480 & 0,00 & 0,175 \\
\hline
\end{tabular}

Nota. $p$ es significativo al nivel $<0,05$

En general, los valores más altos de las diferentes subescalas en las que se hallaron diferencias significativas corresponden al alumnado más joven y la puntuación media va descendiendo según aumenta la edad. Como se ve en la Tabla 5, no existen diferencias significativas entre los dos grupos de alumnado de mayor edad (entre 15-16 años y 17-19 años). En la MI al conocimiento las mayores diferencias se hallaron entre los más jóvenes y los mayores $(p=0,001)$. En el caso de la $M I$ hacia la estimulación y ME identificada se hallaron diferencias entre los de 12-14 años y los de 15-16 años ( $p=0,001)$ y los de 12-14 años y los mayores $(p=0,002)$. Las diferencias más altas se encontraron en la $M E$ de regulación externa, tanto entre los de 12-14 años y 15-16 años $(p<0,000)$ como entre los menores y los de 17-19 años $(p<0,000)$. 
Tabla 5. Pruebas a posteriori según la edad en función de las subescalas del POSQ, LAPOPECQ y SMS

\begin{tabular}{|l|c|c|c|}
\hline Subescalas & $\begin{array}{c}\text { 12-14 años vs. } \\
\text { 15-16 años }\end{array}$ & $\begin{array}{c}\text { 12-14 años vs. } \\
17-19 \text { años }\end{array}$ & $\begin{array}{c}\text { 15-16 años vs. } \\
17-19 \text { años }\end{array}$ \\
\hline Orientación ego & & & \\
\hline Orientación tarea & & & \\
\hline Clima aprendizaje & & & \\
\hline Clima rendimiento & $*$ & $*$ & \\
\hline MI conocimiento & $*$ & $* *$ & \\
\hline MI logro & & & \\
\hline MI estimulación & $* *$ & $*$ & \\
\hline ME identificada & $* *$ & $* *$ & \\
\hline ME introyectada & & & \\
\hline ME regulación externa & $* *$ & $* *$ & \\
\hline Amotivación & & & \\
\hline
\end{tabular}

$* p<0,05 ; * * p<0,01$.

\section{DISCUSIÓN}

El objetivo fundamental de esta investigación ha sido conocer cómo el sexo y la edad del alumnado influye en las orientaciones de meta, la motivación autodeterminada y el clima motivacional en EF. Como se aprecia en las correlaciones, la mayoría de ellas mantienen relaciones significativas con el resto de dimensiones, ello es indicador de la interconexión tan importante que existe entre ambos constructos teóricos. Por tanto, y siguiendo a (Buckworth, Lee, Regan, Schneider \& DiClemente (2006) y Moreno et al. (2008), entre otros, la adecuada combinación de las orientaciones disposicionales y los climas motivacionales son necesarios para avanzar en la mejora de la motivación autodeterminada en el proceso de enseñanza-aprendizaje.

Los datos arrojan resultados bastante positivos, debido a que la mayor parte de la muestra analizada afirma estar orientada a la tarea y percibir un clima tarea. Resultados similares a los encontrados por Moreno, Hellín, Hellín y Cervelló (2006b) y Moreno, Martínez y Cervelló (2011), donde la orientación a la tarea superaba a la del ego, y por Moreno, Llamas y Ruiz (2006c), Moreno, Cervelló, Zomeño, y Marín (2009), donde el clima tarea superaba al clima ego. Estos datos muestran que los alumnos se orientan hacia el aprendizaje, juzgándose más con ellos mismos que con los compañeros, y percibiendo un clima que propicia el interés y la motivación de estos alumnos hacia la asignatura y hacia sus contenidos. Estos datos son de gran interés, pues incluso podrían ayudar a adquirir ciertas actitudes y valores de esfuerzo, sacrificio y trabajo, tan patentes hoy en los sistemas educativos de muchos países.

En cuanto a los resultados obtenidos a partir del SMS, los valores más altos son los que hacen referencia a las distintas dimensiones de la motivación intrínseca, especialmente la motivación intrínseca hacia el logro, aunque también aparece la motivación 
extrínseca introyectada como uno de los valores más altos de la escala. Estos resultados son similares a los encontrados por Moreno et al. (2006c), ya que los mayores valores se encontraron en la motivación intrínseca de conocimiento, de estimulación y de ejecución, seguida de la motivación extrínseca identificada e introyectada. Este patrón, que comienza a ser común, denota el interés del alumnado de estas edades por la asignatura. Por este motivo, los resultados aquí hallados son esperanzadores, ya que se ha demostrado que existe relación directa entre la motivación intrínseca en las clases de EF y el compromiso motor (Standage, Duda \& Ntoumanis, 2003; Ferrer-Caja y Weiss, 2000) e incluso con el deseo de participar en actividades deportivas en el futuro (Sproule et al., 2007; Ntoumanis, 2005; Hein et al., 2004). Por tanto, si el alumno se encuentra motivado, existen altas posibilidades de que realice práctica física en su tiempo libre.

Se puede observar cómo los varones obtienen mayor puntuación en todas las variables, excepto en el clima aprendizaje y en la amotivación, donde no existen diferencias significativas. Estos datos concuerdan por ejemplo con Duda \& Whitehead (1998), Duda (2000) y Moreno et al. (2006b), quienes encontraron también que los varones se encuentran más orientados al ego que las mujeres.

Del mismo modo, también los resultados hallados respecto del clima motivacional percibido por el alumnado son similares a los encontrados por Carr \& Weigand (2001), Cervelló y Santos-Rosa (2000), Martínez-Galindo, Alonso, Cervelló y Moreno (2009) y Jairo y Márquez (2008), en los que se demuestra que los varones presentan un mayor clima rendimiento que las mujeres; y a los realizados por Walling et al. (1993) y Ntoumanis \& Biddle (1999), en donde las mujeres estaban más implicadas en un clima motivacional orientado a la tarea que los varones. Resultados contrarios a los encontrados por Papaioannou y Kouli (1999), White, Kavussanu \& Guest (1998) y Flores, Salguero \& Márquez (2008) donde las mujeres tienden más a percibir un clima aprendizaje que los hombres que lo perciben orientado al rendimiento. No obstante, en los resultados de esta investigación no se encontraron diferencias significativas en el clima aprendizaje. Estos resultados manifiestan la importancia de que el docente trabaje tanto el clima aprendizaje como el clima rendimiento, sabiendo orientar y combinar de manera consciente ambos enfoques, dado que los resultados en chicos y chicas son diferentes.

Respecto de la motivación, aunque no existen diferencias significativas en todas las dimensiones, sí es cierto que siempre los chicos presentan valores mayores a las chicas. Esta tendencia es común en el trabajo de Moreno et al. (2006a), quienes mostraron que los varones se sienten más motivados que las mujeres. No obstante, otros trabajos como los de Moreno et al. (2006b) y Núñez, Martín-Albo y Navarro (2005) concluyen que son las féminas las que tienen valores mayores en la motivación intrínseca, y los chicos en la extrínseca. Esto puede deberse, en parte, al afán por competir, por ser mejor que los demás, más propio de los chicos que de las chicas.

En cuanto a la edad, se ha demostrado que aquellos alumnos más jóvenes son los que presentan los valores más altos de orientación al rendimiento. Esto puede deberse, al cambio tan importante que hay entre la EF recibida en la etapa de primaria y la recibida en la etapa de secundaria, pues los alumnos, cuando acceden a secundaria, encuentran esta asignatura muy cambiante a la vez que divertida y motivante respecto de la de la etapa anterior, donde ellos comienzan a sentirse mayores y quieren competir más. En cambio, en el estudio llevado a cabo por Flores et al. (2008) los estudiantes más jóvenes puntuaron más alto en ambas orientaciones de meta. 
Finalmente, y en cuanto a la motivación, se observa cómo los alumnos más jóvenes son a la vez los alumnos con una motivación más intrínseca, y que conforme se avanza en edad, hay cada vez una motivación más extrínseca y cada vez una menor motivación. Acerca de esto, autores como Moreno et al. (2006a) hallaron que los alumnos de 14-15 años puntuaban más alto en la motivación intrínseca, extrínseca y amotivación, aspecto que en este trabajo no se puede corroborar perfectamente.

Resumiendo, los resultados demuestran la importancia que tiene la figura del profesor en las clases de EF en esta etapa educativa, tanto en la creación de un clima acorde a los estudiantes, como en la influencia en sus orientaciones y motivaciones. Desde un punto de vista práctico y teniendo en cuenta los paradigmas y corriente de la EF (Moreno, Campos y Almonacid, 2012), el profesor debe diseñar sus clases con un clima motivacional orientado a la tarea, favoreciendo un entorno ideal para la mejora del aprendizaje y el incremento del compromiso de los alumnos en las clases de EF.

\subsection{LIMITACIONES Y FORTALEZAS}

En esta investigación se puede indicar que una de las limitaciones ha sido no haber realizado un diseño muestral con representatividad de la población estudiada. Las limitaciones económicas y temporales llevaron a desarrollar el estudio mediante muestra seleccionada por conveniencia. No obstante, el tamaño de la muestra es una fortaleza a destacar del presente trabajo. Otra de las limitaciones que se presenta en este tipo de investigaciones va referida al momento en el que se encuesta a los estudiantes, ya que durante la recogida de datos en cada centro y nivel se están impartiendo contenidos diferentes. Una opción de cara a futuros estudios es que se tenga en cuenta que el alumnado encuestado esté recibiendo los mismos contenidos y precisar, así, mejor el análisis de estas variables.

\section{REFERENCIAS BIBLIOGRÁFICAS}

Ames, C. (1992a). Classrooms, goals, structures, and student motivation. Journal of Educational Psychology, vol. 84 (3), 261-271.

(1992b). Achievement goals, motivational climate, and motivational processes. In G. C. Roberts (Ed.), Motivation in sport and exercise (pp. 161-176). Champaign, IL: Human Kinetics.

Bentler, P. M. (2007). On tests and indices for evaluating structural models. Personality and Individual Differences, vol. 42 (5), 825-829.

Bollen, K. \& Long, J. S. (1994). Testing structural equation models. Newbury Park, CA: Sage.

Brière, N., Vallerand, R., Blais, N. et Pelletier, L. (1995). Développement et validation d'une mesure de motivation intrinsèque, extrinsèque et d'amotivation en contexte sportif: L'Échelle de Motivation dans les Sports (ÉMS). International Journal of Sport Psychology, vol. 26 (4), 465-489.

Buckworth, J., Lee, R. E., Regan, G., Schneider, L. K. \& DiClemente, C. C. (2006). Decomposing intrinsic and extrinsic motivation for exercise: Application to stages of motivational readiness. Psychology of Sport and Exercise, vol. 8 (4), 441-461.

Carr, S. \& Weigand, D. A. (2001). Parental, peer, teacher and sporting hero influence on the goal orientations of children in physical education. European Physical Education Review, vol. 7 (3), 305-328.

Castillo, I., Balaguer, I. y Duda, J. L. (2000). Las orientaciones de meta y los motivos de práctica deportiva en los jóvenes deportistas valencianos escolarizados. Revista de Psicología del Deporte, vol. 9 (1-2), 37-50. 
Cervelló, E., Jiménez, R., Fenoll, A., Ramos, L., Del Villar, F. \& Santos-Rosa, F. J. (2002). A social-cognitive approach to the study of coeducation and discipline in Physical Education classes. SOCIOTAM, Revista Internacional de Ciencias Sociales y Humanidades, vol. 11, 43-64.

y Santos-Rosa, F. J. (2000). Motivación en las clases de EF: Un estudio de la perspectiva de las metas de logro en el contexto educativo. Revista de Psicología del Deporte, vol. 9 (1-2), 51-70. Escartí, A. y Balagué, G. (1999). Relaciones entre la orientación de meta disposicional y la satisfacción con los resultados deportivos, las creencias sobre las causas de éxito en el deporte y la diversión con la práctica deportiva. Revista de Psicología del Deporte, vol. 8 (1), 7-21.

Deci, E. L. \& Ryan, R. M. (2000). The "what" and "why" of goal pursuits: Human needs and the self-determination of behavior. Psychological Inquiry, vol. 11 (4), 227-268.

(1985). Intrinsic motivation and self-determination in human behavior. New York: Plenum.

Duda, J. L. \& Whitehead, J. (1998). Measurement of goal perspectives in the physical domain. In J. L. Duda (Ed.), Advances in sport and exercise psychology measurement (pp. 21-48). Michigan: Fitness Information Techonology, Inc.

\& Nicholls, J. G. (1992). Dimensions of achievement motivation in schoolwork and sport. Journal of Education Psychology, vol. 84 (3), 290-299.

Ferrer-Caja, E. \& Weiss, M. R. (2000). Predictors of intrinsic motivation among adolescent students in physical education. Research Quarterly for Exercise and Sport, vol. 71 (3), 267-279.

Flores, J., Salguero, A. \& Márquez, S. (2008). Goal orientations and perceptions of the motivational climate in physical education classes among Colombian students. Teaching and Teacher Education, vol. 24 (6), 1441-1449.

González-Cutre, D., Sicilia, A. y Moreno, J. A. (2011). Un estudio cuasi-experimental de los efectos del clima motivador tarea en las clases de educación física. Revista de Educación, vol. 356, 677-700.

Granero-Gallegos, A., Baena-Extremera, A., Gómez-López, M. y Abraldes, J. A. (2014). Estudio psicométrico y predicción de la importancia de la Educación Física a partir de las orientaciones de meta ("Perception of Success Questionnaire - POSQ"). Psicologia: Reflexão e Crítica, vol. 27 (3), 443-451. (2013). Análisis preliminar exploratorio del "Sport Motivation Scale (SMS)" adaptado a la Educación Física. Espiral. Cuadernos del profesorado, vol. 6 (12), 3-14.

Hair, J. F., Black, W. C., Babin, B. J. \& Anderson, R. E. (2009). Multivariate data analysis ( $7^{\text {th }}$ Ed.). New York: Pearson Prentice Hall.

Hein, V., Müür, M. \& Koka, A. (2004). Intention to be physically active after school graduation and its relationship to three types of intrinsic motivation. European Physical Education Review, vol. 10 (1), 5-19.

Hooper, D., Coughlan, J. \& Mullen, M. (2008). Structural equation modelling: Guidelines for determining model fit. Electronic Journal of Business Research Methods, vol. 6 (1), 53-60.

$\mathrm{Hu}, \mathrm{L} . \&$ Bentler, P. M. (1999). Cutoff criteria for fit indexes in covariance structure analysis: Conventional criteria versus new alternatives. Structural Equation Modelling, vol. 6 (1), 1-55.

Jairo, A. S. y Márquez, S. (2008). Relación de género, curso y tipo de colegio con el clima motivacional percibido en la Educación Física escolar en estudiantes colombianos. Revista de Educación, vol. 347, 203-227.

Jiménez, R., Cervelló, E., García, T., Santos, F. J. e Iglesias, D. (2007). Estudio de las relaciones entre motivación, práctica deportiva extraescolar y hábitos alimenticios y de descanso en estudiantes de Educación Física. International Journal of Clinical and Health Psychology, vol. 7 (2), 385-401.

Jöreskog, K. G. \& Sörbom, D. (1993). Structural equation modeling with the SIMPLIS command language. Chicago: Scientific Software International.

Kline, R. B. (2005). Principles and practice of structural equation modelling ( $2^{\text {nd }}$ Ed.). New York: The Guilford Press.

Llanos, C., Cervelló, E. y Tabernero, B. (2008). Una investigación sobre el clima motivacional en las clases de Educación Física: Un elemento del entorno a considerar por el profesor. Bordón, vol. 60 (1), 59-76. 
Martínez-Galindo, C., Alonso, N., Cervelló, E. y Moreno, J. A. (2009). Perfiles motivacionales y disciplina en clases de Educación Física. Diferencias según las razones del alumnado para ser disciplinado y la percepción del trato generado por el profesorado en el aula. Cultura y Educación, vol. 21 (3), 331-343.

Moreno, A., Trigueros, C. y Rivera, E. (2013). Autoevaluación y emociones en la formación inicial de profesores de Educación Física. Estudios Pedagógicos, vol. 39 (1), 165-177.

. Campso, M. y Almonacid, A. (2012). Las funciones de la educación física escolar: Una mirada centrada en la justicia social y la reconstrucción del conocimiento. Estudios Pedagógicos, vol. 38 (Número Especial), 13-26.

Moreno, J. A., Zomeño, T. E., Marín, L. M., Ruiz, L. M. y Cervelló, E. (2013). Percepción de la utilidad e importancia de la EF según la motivación generada por el docente. Revista de Educación, vol. 362, 380-401.

. Martínez, C. y Cervelló, E. (2011). Relación predictiva entre la percepción del alumnado de las estrategias de disciplina del profesor y la percepción del trato de igualdad-discriminación en las clases de Educación Física. Revista de Educación, vol. 355, 381-403.

Cervelló, E., Zomeño, T. E. y Marín, L. M. (2009). Predicción de las razones de disciplina en Educación Física. Acción Psicológica, vol. 6 (2), 7-15.

Conte, L., Hellín, P., Hellín, G., Vera, J. A. y Cervelló, E. (2008). Predicción de la motivación autodeterminada según las estrategias para mantener la disciplina y la orientación motivacional en estudiantes adolescentes de educación física. Apuntes de Psicología, vol. 26 (3), 501-516.

. Cervelló, E. y González-Cutre, D. (2006a). Motivación autodeterminada y flujo disposicional en el deporte. Anales de Psicología, vol. 22 (2), 310-317.

Hellín, P., Hellín, G. y Cervelló, E. (2006b). Efectos del género, la edad y la práctica físico-deportiva en las estrategias de disciplina, la orientación disposicional y la motivación autodeterminada en estudiantes adolescentes de Educación Física. En A. Díaz (Ed.), VI Congreso Internacional de Educación Física e Interculturalidad (pp. 1-14). Murcia: ICD.

. Llamas, L. S. y Ruiz, L. M. (2006c). Perfiles motivacionales y su relación con la importancia concedida a la Educación Física. Psicología Educativa, vol. 12 (1), 49-63.

Vera, J. A. y Cervelló, E. (2006d). Evaluación participativa y responsabilidad en Educación Física. Revista de Educación, vol. 340, 731-754.

Nicholls. J. G. (1989). The competitive ethos and democratic education. Cambridge: Harvard University Press.

Ntoumanis, N. (2005). A prospective study of participation in optional school Physical Education using a self-determination theory framework. Journal of Educational Psychology, vol. 97 (3), 444-453. . \& Biddle, S. (1999). Affect and achievements goals in physical activity. A meta-analysis. Scandinavian Journal of Medicine and Science in Sports, vol. 9 (6), 315-332.

Núñez, J. L., Martín-Albo, J., Navarro, J. G. \& González, V. M. (2006). Preliminary validation of a Spanish version of the Sport Motivation Scale. Perceptual and Motor Skills, vol. 102 (3), 919-930. (2005). Validación de la versión española de la Échelle de Motivation en Éducation. Psicothema, vol. 17 (2), 344-349.

Papaioannou, A. \& Kouli, O. (1999). The effects of task structure, perceived motivational climate, and goal orientation on students' task involvement and anxiety. Journal of Applied Sport Psychology, vol. 11 (1), 51-71.

. (1994).The development of a questionnaire to measure achievement orientations in Physical Education. Research Quarterly for Exercise and Sport, vol. 65 (1), 11-20.

Pelletier, L. G., Fortier, M. S., Vallerand, R. J., Tuson, K. M., Brière, N. M. \& Blais, M. R. (1995a). Toward a new measure of intrinsic motivation, extrinsic motivation, and amotivation in sports: The Sport Motivation Scale (SMS). Journal of Sport and Exercise Psychology, vol. 17 (1), 35-53. 
Vallerand, R. J., Green-Demers, I., Brière, N. M. \& Blais, M. R. (1995b). Leisure and mental health: Relationship between leisure involvement and psychological well-being. Canadian Journal of Behavioral Science, vol. 27, 214-225.

Roberts, G. C. (2001). Understanding the dynamics of motivation in physical activity: The influence of achievement goals on motivational process. In G. C. Roberts (Ed.), Advances in motivation in sport and exercise (pp. 1-50). Champaign, IL: Human Kinetics.

Treasure, D. C. \& Balagué, G. (1998). Achievement goals in sport: The development and validation of the Perception of Success Questionnaire. Journal of Sport Sciences, vol. 16 (4), 337-347.

. \& Balagué, G. (1991). The development and validation of the Perception of Success Questionnaire. $8^{\text {th }}$ European FEPSAC Congress. Cologne, Germany.

Ryan, R. M. \& Deci, E. L. (2000). Self-determination theory and the facilitation of intrinsic motivation. Social development and well-being. American Psychologist, vol. 55 (1), 68-78.

Sproule, J., Wang, C. K. J., Morgan, K., McNeill, M. \& McMorris, T. (2007). Effects of motivational climate in Singaporean Physical Education lessons on intrinsic motivation and physical activity intention. Personality and Individual Differences, vol. 43 (5), 1037-1049.

Standage, M., Duda, J. L. \& Ntoumanis, N. (2003). A model of contextual motivation in Physical Education: Using constructs from self-determination and achievement goal theories to predict physical activity intentions. Journal of Educational Psychology, vol. 95 (1), 97-110.

Tabachnick, B. G. \& Fidell, S. A. (2006). Using multivariate statistics (5 ${ }^{\text {th }}$ Ed.). Boston: Allyn \& Bacon.

Taylor, I. M., Ntoumanis, N. \& Standage, M. (2008). A self-determination theory approach to understanding the antecedents of teachers' motivational strategies in Physical Education. Journal of Sport and Exercise Psychology, vol. 30 (1), 75-94.

Trudeau, F., Laurencelle, L., Tremblay, J., Rajic, M. \& Shephard, R. J. (1999). Daily primary school Physical Education: Effects on physical activity during adult life. Medicine and Science in Sports and Exercise, vol. 31 (1), 111-117.

Vallerand, R. J., Blais, M. R., Brière, N. M. et Pelletier, L. G. (1989). Construction et validation de l'Échelle de Motivation en Éducation (EME). Canadian Journal of Behavioral Science, vol. 21 (3), 323-349.

Walling, M., Duda, J. L. \& Chi, L. (1993). The perceived motivational climate in sport questionnaire: Construct and predictive validity. The Journal of Sport and Exercise Psychology, vol. 15 (2), 172-183.

White, S. A., Kavussanu, M. \& Guest, S. M. (1998). Goal orientations and perceptions of the motivational climate created by significant others. European Journal of Physical Education, vol. 3 (2), 212-228. 
